\title{
Efecto filtrador del pepino de mar sobre la materia orgánica en cultivos de tilapia gris
}

\author{
Filter effect of sea cucumber on organic matter in gray tilapia crops
}

\author{
Carmen Isabel Hernández Rivera ${ }^{1}$ \\ Noelia Erlinda Cea Navas ${ }^{2}$ \\ Alixon Enrique Pacheco Interiano ${ }^{3}$ \\ Hermes Ramón Reyes Guido ${ }^{4}$
}

\section{Resumen}

La acuicultura puede ser delimitada como una acción o rubro mercantil alternativo, en cuanto a la crianza de recursos hidrobiológicos, denominados de igual manera como peces, crustáceos, moluscos y vegetación acuática, en ambientes totalmente discordantes en relación a los naturales, en donde las variables físicas y químicas pueden ser relativamente controlables, con el objetivo de sustituir y perfeccionar las condiciones mediante las cuales estos organismos puedan satisfacer sus necesidades como si estuvieran en ambientes particularmente normales. La acuicultura a nivel mundial figura como una de las actividades con mayor utilización de los recursos hídricos en el cual se debe apostar por la implementación de sistemas que supongan un ahorro de agua. Este estudio fue realizado en las instalaciones del Laboratorio de Investigaciones Marinas y Acuícolas (LIMA) de la UNAN-León, con la intención de comparar dos diferentes sistemas de cultivo (Monocultivo y Policultivo), que permitieran evaluar la capacidad de filtración del pepino de mar (Isostichopus fuscus) sobre la materia orgánica producida en cultivos de tilapia gris (Oreochromis sp), obteniendo como resultados de la investigación $453.0 \mathrm{mg} / \mathrm{Lo2}$ de materia orgánica, $0.32 \mathrm{mg} / \mathrm{Lt}$ de materia en suspensión, 6.29 mgNH3-N/L (Nitrógeno Amoniacal), 43.35 mg No2 -IL (Nitrito) y $18.4 \mathrm{mg}$ No3/L (Nitrato) para el sistema de policultivo; por otro lado se obtuvieron datos de $512 \mathrm{mg} / \mathrm{Lo2}$ de materia orgánica, $4.48 \mathrm{mg} / \mathrm{Lt}$ de materia en suspensión, 17.02 mgNH3-N/L, 26.90 mg No2 -IL, 33.87 mg NO3/L para el sistema de monocultivo, denotando así la efectividad del pepino de mar (Isostichopus fuscus) sobre el objeto de estudio.

Palabras clave: Tilapia; policultivo; pepino de mar.

\footnotetext{
1 Master en desarrollo local sostenible, Profesora de la Escuela de Ciencias Agrarias y Veterinarias de la Universidad Nacional Autónoma de Nicaragua-León. Correo: carmen.hernandez@ev.unanleon.edu.ni; No. ORCID https://orcid.org/o0oo-0002-3002-5255

2 Licenciada en estadística. Profesora de la Escuela de Ciencias Agrarias y Veterinarias de la Universidad Nacional Autónoma de Nicaragua- León. Correo: noelia.cea@ev.unanleon.edu.ni; No. ORCID: https://orcid.org/oooo-ooo1-7913-9459

3 Ingeniero Acuícola correo: pachec02996@gmail.com ORCID https://orcid.org/oooo-ooo2-6091-9268

4 Mas Ingeniero Acuícola. Correo: hreyesguido@yahoo.com ORCID https://orcid.org/oooo-00o2-4080-9168
}

Recibido: 22/02/2019 Aprobado: 03/06/2019 


\section{Abstract}

Aquaculture can be defined as an action or alternative commercial item, in terms of raising hydrobiological resources, similarly called fish, crustaceans, molluscs and aquatic vegetation, in environments totally discordant in relation to natural ones, where the variables physical and chemical can be relatively controllable, with the aim of replacing and improving the conditions by which these organisms can meet their needs as if they were in particularly normal environments. Worldwide aquaculture is one of the activities with the greatest use of water resources, in which it is necessary to bet on the implementation of systems that suppose water savings. This study was conducted in the Marine and Aquaculture Research Laboratory (LIMA) facilities of the UNAN-León, with the intention of comparing two different farming systems (monoculture and polyculture), which would allow to evaluate the filtration capacity of sea cucumber. (Isostichopus fuscus) on the organic matter produced in gray tilapia cultures (Oreochromis niloticus), obtaining as a result of the research $453.0 \mathrm{mg} /$ Lo2 of organic matter, $0.32 \mathrm{mg}$ / Lt of suspended matter, $6.29 \mathrm{mgNH}_{3}-\mathrm{N}$ / L (Nitrogen Ammoniacal), 43.35 mg No2 -IL (Nitrite) and 18.4 mg No3 / L (Nitrate) for the polyculture system; On the other hand, data were obtained of $512 \mathrm{mg} / \mathrm{Lo2}$ of organic matter, $4.48 \mathrm{mg} / \mathrm{Lt}$ of suspended matter, $17.02 \mathrm{mgNH}_{3}-\mathrm{N} / \mathrm{L}, 26.90 \mathrm{mg}$ No2 -IL, $33.87 \mathrm{mg} \mathrm{NO}_{3}$ / L for the monoculture system, denoting the effectiveness of sea cucumber (Isostichopus fuscus) on the object of study.

Keywords: Tilapia; polyculture; sea cucumber.

\section{Introducción}

Las acciones humanas han sido siempre los motivos principales que han originado que un bien o recurso natural sufra cambios negativos. Por lo que en la actualidad se debe de hablar de una acuicultura que pueda perdurar en el tiempo, sin ser causa alguna para el deterioro del ambiente. Por lo tanto, una acuicultura responsable viene a ser aquella que además de ser aprovechada, se hace conscientemente.

El reto de la acuicultura, como una industria en constante desarrollo, principalmente se centra en conseguir los usufructos económicos y sociales sin comprometer la estabilidad del medio, siendo necesarios de alguna manera estudios eficientes meticulosamente planeados que permitan corroborar que esta es una opción de producción con amplias probabilidades de ser llevada a cabo de manera sostenible (Sandra Pardo, 2006).

Una de las mayores problemáticas en la producción acuícola se debe a la acumulación e incremento de materiales sueltos, producidos principalmente por las excreciones de los organismos en cultivo, por las prácticas de alimentación y por otros insumos que son adicionados en los estanques de cultivo (Tacon \& Forster, 2003). Por lo que 
se plantea como un tratamiento alternativo para la reducción de los productos generados en un estanque de cultivo de peces los sistemas de monocultivo con pepinos de mar, para incentivar el aprovechamiento de los residuos que no son aprovechados por los peces.

\section{Revisión de literatura}

La acuicultura al igual que cualquier industria, genera impactos ambientales negativos, ya que es desarrollada en ecosistemas artificiales diferentes a los naturales, afectando de manera directa a los distintos cuerpos de agua, debido a la cantidad de efluentes ricos en materia orgánica que son vertidos al medio sin un previo tratamiento (Sandra Pardo, 2006). De acuerdo con lo descrito por Mortera (2013), se puede afirmar que una de las mayores problemáticas ambientales originadas por la práctica acuícola, ha sido la descarga directa de los efluentes con gran contenido de detritus y nutrientes como Fósforo y Nitrógeno, para lo cual es necesario la implementación de tratamientos alternativos que permitan reducir el grado de eutrofización en los cuerpos receptores.

En relación a los organismos que se cultivan, la acuicultura como tal, se ha dividido en varios tipos, siendo la piscicultura uno de los más desarrollados dentro de la industria, encabezando el cultivo de tilapia como el producto de mayor importancia, por lo tanto, el desenvolvimiento de una piscicultura más limpia, se vuelve cada vez más conveniente para el fortalecimiento de la industria y la protección del medio ambiente. La piscicultura tiene por objetivo, la producción de peces para el consumo humano ya que un elevado porcentaje de la población humana a nivel mundial, ha sido y sigue siendo mal nutrida, debido a la escasez de fuentes baratas de proteína animal, por lo que se ha considerado encontrar fuentes alternativas de consumo que suplan las necesidades alimenticias a las cuales el hombre se ha enfrentado. Los peces representan una fuente alimenticia de alta calidad, por lo que la piscicultura se presenta como una fuente de proteína animal que sirve para mejorar las dietas, y fuentes de ingresos que contribuyan al mejoramiento de la economía familiar (Citado por Meyer, 2004).

Pese a que todavía no se registran datos, a nivel nacional, concernientes a la investigación de sistemas de policultivo entre tilapias y pepinos de mar, se presentan algunas referencias similares que abordan la misma problemática. La mayoría de los estudios que se han desarrollado en este sentido, son con moluscos de agua salada, tal es el caso del sururu de mangle Mytella guyanensis y de la ostra Crassotrea rhizophorae probados en la biorremediación de los impactos ocasionados por los efluentes de la camaronicultura en el nororiente brasileiro. (Olivera \& Brito., 2005). Relacionado a los moluscos de agua dulce se hallan algunas referencias en Europa (Reeders, Vaate, \& J.Slim., 1989), y en lagos de América del norte con Dreissenia polymorpha, presentándose como una excelente filtradora capaz de aumentar la transparencia y disminuir la clorofila después de su inclusión (Bunt, Mac lsaac, \& Sprules, 1993). 
Un trabajo con énfasis en la mitigación de la eutrofización en estanques de cultivo de salmón fue llevado a cabo en Chile, con Diplodon Chilensis, convirtiendo estanques cerrados hiper-eutróficos en estanques oligotróficos. La capacidad filtradora del molusco declinó después de 60 días, necesitándose una mayor investigación al respecto (Soto \& Mena Guillermo, 1999).

\section{Materiales y métodos}

Estainvestigación fue realizada en el período de junio a octubre del 2017, en las instalaciones del Laboratorio de Investigaciones Marinas y Acuícolas (LIMA), ubicado en la localidad de Las Penitas, León- Nicaragua, la metodología utilizada se basó en una investigación del tipo pre-experimental o correlacional.

La población de estudio fue el Pepino de mar de la especie Isostichopus Fuscus y tilapia gris (Oreochromis niloticus), la cual fue cultivada en el dispositivo experimental desde un peso de $1.77 \mathrm{gr}$, el tamaño de la muestra fue de 3 pepinos de mar/m3 y de 10 tilapias/m3. Dentro del procedimiento de siembra de tilapia en agua salobre, el período de aclimatación implica el incremento gradual de la salinidad hasta alcanzar la que tiene el dispositivo receptor (USAID, 2007). En el caso del pepino de mar, se capturaron en las aguas del Pacífico de Nicaragua y una vez capturados fueron trasladados al sitio de estudio, en donde se dejaron reposar por 15 minutos para evitar someterlos a un grado mayor de estrés una vez pasado ese tiempo, se procedió en bolsas a la tina en donde serían depositados para dar inicio al estudio (Peralta, 2010).

Para la alimentación de nuestros organismos en cultivo (Tilapia gris), se hizo uso de alimento pelletizado comercial al $28 \%$ proteínas, las raciones alimenticias fueron divididas en cuatro dietas durante el día, las cuales se distribuyeron de la siguiente manera: $25 \%$ a las 8 de la mañana y $25 \%$ a las 11 de la mañana; el $50 \%$ restante fue distribuido a las 2 y 4 de la tarde, dividiendo la dieta en partes iguales. (Pronagro, 2002). Por otra parte durante el estudio se monitoreo oxígeno disuelto, temperatura, salinidad, pH y turbidez. La turbidez que se tomó a las $11 \mathrm{am}$, los datos de los otros parámetros físico químico se recolectaron dos veces al día a las 6 am y a las 6 pm. (M. Pereira, Conversación personal, 14 de mayo de 2017).

Para medir los niveles de materia orgánica que presenta el dispositivo experimental se utilizó el instrumento denominado Cono de Imhoff, se tomó una muestra de $1 \mathrm{~L}$ de agua, introduciendo el cono al dispositivo hasta la altura del hombro para poder tomar la muestra del fondo a la superficie de la columna de agua, la muestra tomada se dejó sedimentar durante 45 minutos, posterior a ello fueron removidas la paredes del cono mediante una varilla o rotación y se dejó reposar durante 15 minutos más, se registró el volumen de solidos sedimentados en la parte inferior del cono. 
Para realizar el recuento de micro algas se hizo uso de dos tipos de cámaras de recuento, la cámara Neubauer y cámara Sedgwick-Rafter, en la primera se contabilizaron celular de hasta 50 micras y en la segunda células que hacienden a las 500 micras, esto permitía tener un conteo más apegado a la realidad de cada dispositivo. Para poder determinar la diversidad de especies de micro algas presente en el dispositivo experimental se usó índice de Shannon-Weaver, se usa en ecología para poder medir la diversidad específica de especies.

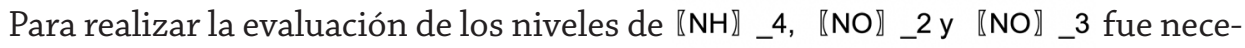
sario hacer un análisis químico al agua de del dispositivo, tomando una muestra de agua de los mismos, la cual fue trasladada al Laboratorio de Química del agua, ubicado en el Departamento de Química de la Facultad de Ciencias y Tecnologías (UNAN), en la ciudad de León, en donde se realizaron los respectivos análisis, estos fueron realizados cada 30 días partiendo del primer día de cultivo.

Al finalizar para realizar el análisis de los datos recolectados de esta investigación, se hizo uso del programa llamado, SPSS versión 23.

Resultados y discusión (Letra Arial 12, Izquierda justificada interlineado

\section{Oxígeno disuelto}

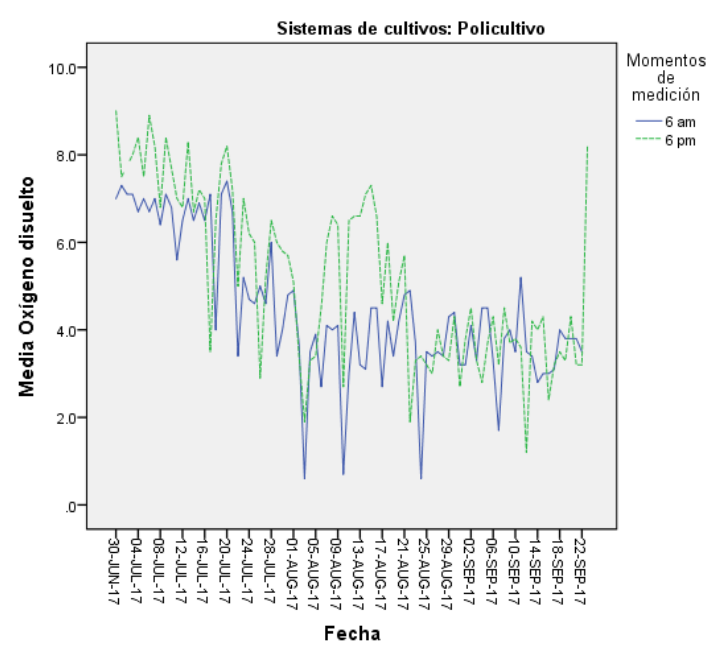

Figura No. 1: Promedio de Oxígeno medido en el sistema de policultivo.

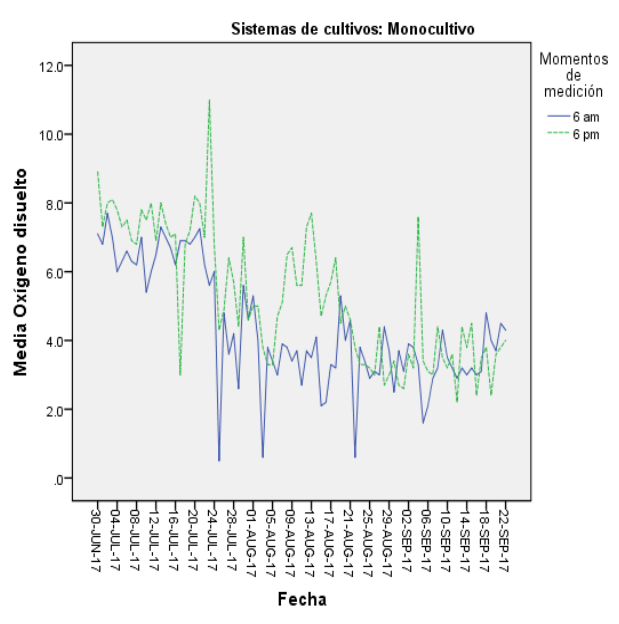

Figura No. 2: Promedio de oxigeno medido en el sistema de Monocultivo

Basado en la figura 1, el oxígeno disuelto presentó una amplia variabilidad a lo largo del cultivo, entre $9.3 \mathrm{mg} / \mathrm{lt}$ y $0.64 \mathrm{mg} / \mathrm{lt}$. 
Aunque Peralta, (2010) señala un oxígeno optimo de $7.0 \mathrm{mg} / \mathrm{lt}$ para el cultivo y desarrollo del pepino de mar (Isostichopus fuscus) y Pérez, Bejarano, \& Barragan, (2013) muestra un oxigeno mayor a $4.5 \mathrm{mg} / \mathrm{lt}$ para el cultivo de Tilapia Gris (Oreochromis niloticus) no se presento ningun tipo de afectacion en los organismos cultivados pese al inestable comportamiento del oxígeno disuelto presente en el dispositivo.

En base a la figura 2: el comportamiento del oxígeno disuelto en el sistema de monocultivo monitoreado en dos momentos del día, presentó un máximo $11.5 \mathrm{mg} / \mathrm{lt}$ y un mínimo de $0.6 \mathrm{mg} / \mathrm{lt}$ con un valor promedio de $3 \mathrm{mg} / \mathrm{lt}$ por la mañana y cercano a $6 \mathrm{mg} / \mathrm{lt}$ por la tarde.

Según Pérez, Bejarano, \& Barragan, (2013), el oxígeno disuelto óptimo para el cultivo de Tilapia Gris (Oreochromis niloticus), debe ser mayor a $4.5 \mathrm{mg} / \mathrm{lt}$ de concentración, y asegura que valores menores a este dato pueden ser nocivos para los organismos cultivados, sin embargo, en este sistema de cultivo se muestran valores menores, y se confirma que no se presentan afectaciones a exposiciones bajas de oxigenos en periodos relativamente cortos, los cueles no repercutieron de manera negativa en el desarrollo y crecimiento de los organismos que fueron cultivados.

\section{Temperatura}

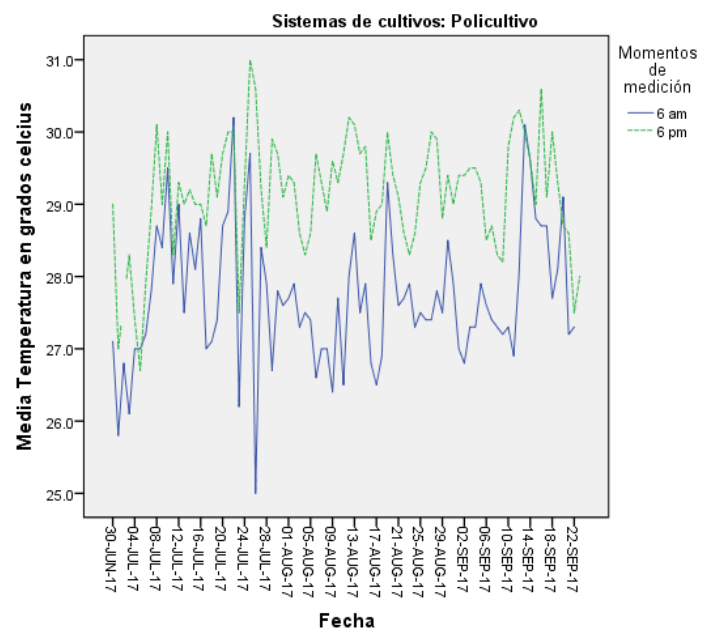

Figura No. 3: Datos de temperaturas en grados Celsius tomados en el sistema de policultivo.

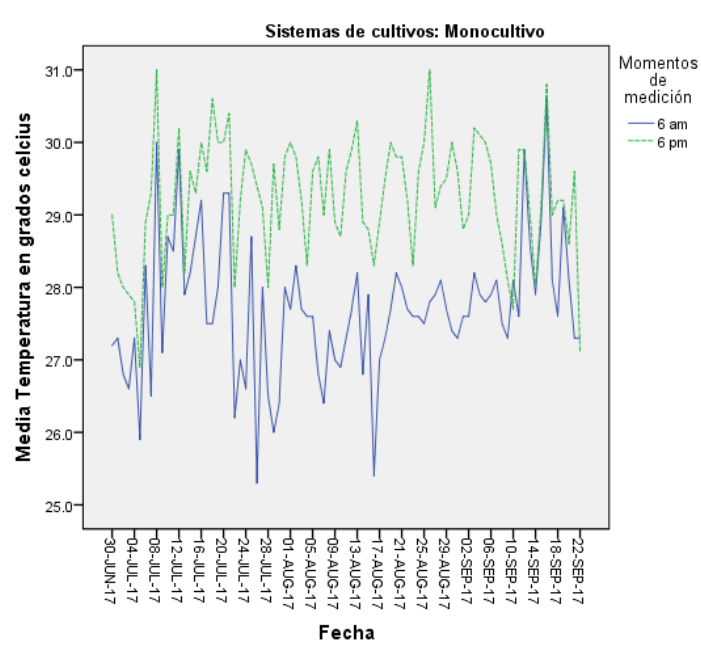

Figura No. 4: Datos de temperaturas en Grados Celsius tomados en sistema de Monocultivo.

Respecto a la figura 3, el parámetro de temperatura monitoreado en dos momentos del día en el dispositivo experimental de policultivo muestra un promedio de $28^{\circ} \mathrm{C}$, con un máximo de $31^{\circ} \mathrm{C}$ y un mínimo de $25^{\circ} \mathrm{C}$, encontrándose dentro de los rangos óptimos, tanto para el crecimiento y desarrollo del pepino de mar (Isostichopus fuscus), como 
para la Tilapia Gris (Oreochromis niloticus), mostrandose esta dispersión en los datos por la inestabilidad del clima durante el periodo de cultivo (Junio-Octubre 2017).

Acorde a la figura 4, el comportamiento de la temperatura en el dispositivo de monocultivo tuvo una amplia dispersión entre los dos momentos de monitoreo, encontrándose a si con una media de $27.5^{\circ} \mathrm{C}$ a las 6: am y de $29.5^{\circ} \mathrm{C}$ a las 6: pm, encontrándose los datos dentro de los rangos recomendados por NICOVITA, (2002) para el cultivo de Tilapia Gris (Oreochromis niloticus), asociando esta varianza al comportamiento del clima durante el tiempo de cultivo.

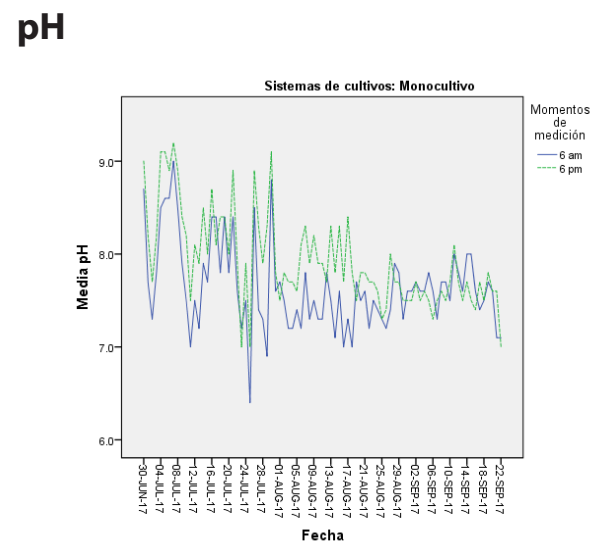

Figura N0. 5: Potencial de iones de hidrógeno tomados en sistema de policultivo.

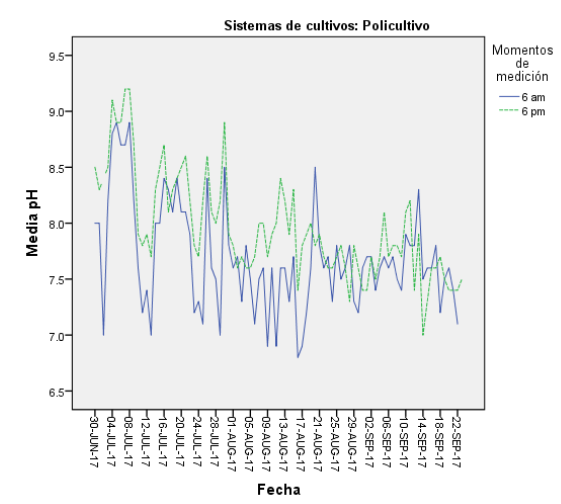

Figura No. 6: Potencial de iones de hidrógenos tomados en el sistema de monocultivo.

En base a la figura 5, la variabilidad de $\mathrm{pH}$ evaluado en el sistema de policultivo, se encuentra con similitudes entre los momentos de monitoreo ( 6 am y $6 \mathrm{pm}$ ), pero amplia dispersión entre valores, dando así una media de 7.5, un máximo de 9.3 y un mínimo de 6.4. Manteniéndose de esta manera dentro de los rangos óptimos propuestos por (Peralta, 2010) para el pepino de mar (Isostichopus fuscus), y (FONDEPES, 2004) para el cultivo de la Tilapia Gris (Oreochromis sp).

En relación con la figura 6, el pH muestra mayor tasa de variabilidad en las primeras semanas de cultivo, en ambos momentos de monitoreo (6: am y 6: pm), posteriormente los valores se encuentran cercanos a la media (7.5), siendo así un máximo de 9.2 y un mínimo de 6.4 para ambos momentos.

Dado esto, se puede señalar que este parámetro se encontró dentro de los rangos requeridos para el cultivo de la Tilapia Gris (Oreochromis sp) en conformidad con lo descrito por (Boyd, 1996), quien asegura que exposiciones bajas de $\mathrm{pH}$ (menores a 5) puede provocar estrés por acidez a los organismos en cultivo. 
Dicha variabilidad en las primeras semanas de cultivo es atribuida al proceso de aclimatación, donde los organismos liberan excretas que contienen elementos nitrogenados que influyen directamente sobre el $\mathrm{pH}$ del agua.

\section{Salinidad}

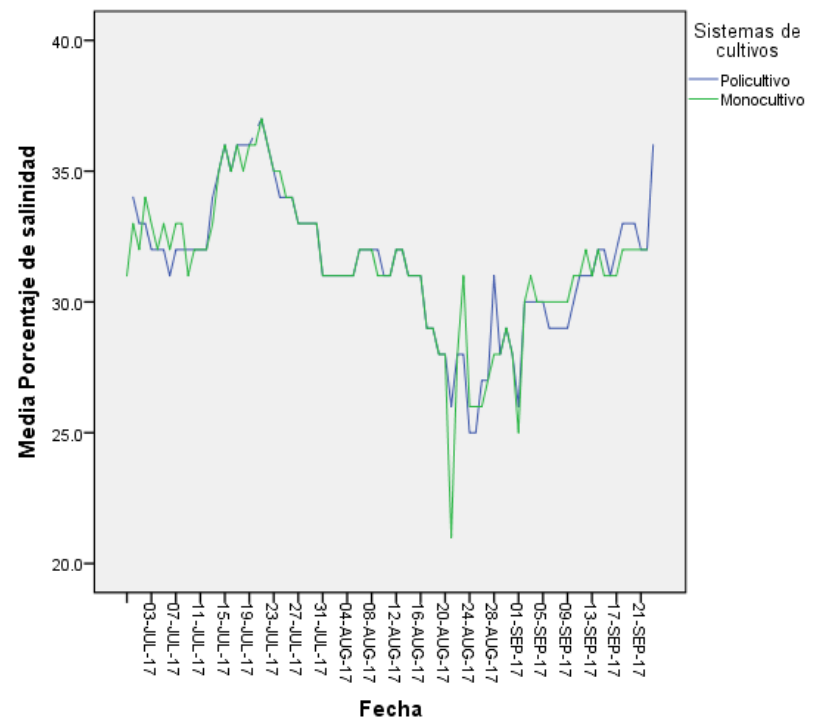

Figura No. 7: Datos de iones de sal, tomados en los sistemas de policultivo y monocultivo.

Los datos de salinidad tuvieron comportamientos similares en ambos sistemas de cultivo (policultivo y monocultivo), con una dispersión entre 22 y 36 ppm, con valores por encima de los $30 \mathrm{ppm}$ dentro de las primeras semanas de cultivo, mientras que para las siguientes semanas predominaron valores por debajo de los $30 \mathrm{ppm}$ hasta los $33 \mathrm{ppm}$. Prevaleciendo así dentro de los rangos óptimos señalados por FONDEPES (Coat., 2004), para la tilapia gris (Oreochromis sp) y (Peralta, 2010), para el pepino de mar (Isostichopus fuscus)

\section{Turbidez}

Dado los valores presentes en la figura(8), la turbidez encontrada en el sistema de policultivo tuvo menor dispersión con respecto al sistema de monocultivo, siendo este el que muestra un valor máximo de $78 \mathrm{~cm}$ de profundidad y un mínimo de 32 $\mathrm{cm}$, mientras que el policultivo presento un valor máximo de $70 \mathrm{~cm}$ y mínimo de 30 $\mathrm{cm}$ de profundidad, siendo atribuido dicha diferencia a la presencia de organismos filtradores dentro del estanque de policultivo los cuáles de acuerdo a (Ruiz, Ibáñez, \& Cacerez, 2007) presentan características alimenticias inclinadas principalmente al consumo de micro algas, lo que permitió cantidades casi estables de concentración Fito planctónica sobre la columna de agua, mientras que en el monocultivo se presume un 
ciclo ininterrumpido de la curva de crecimiento del fitoplancton lo cual permitía que por ciertos periodos el estanque estuviese con menor presencia de algas al finalizar su estado de latencia.

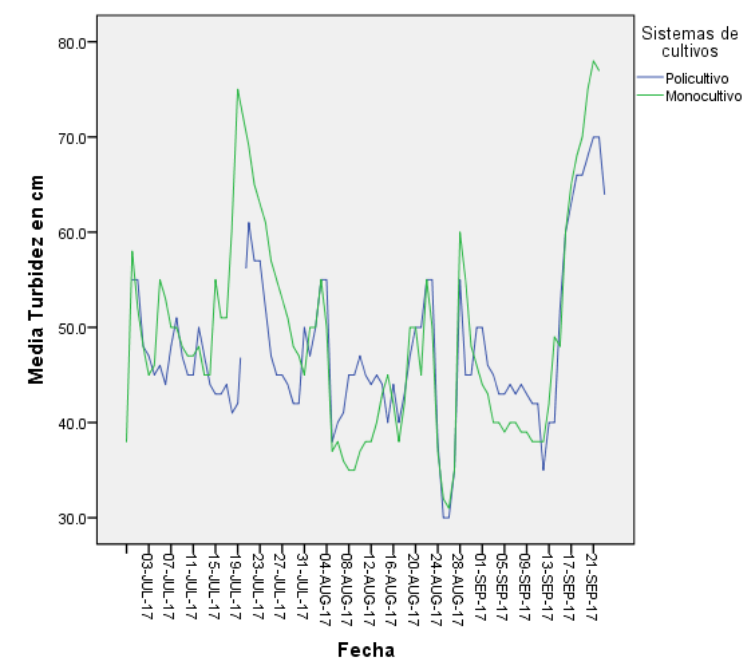

Figura No. 8: Datos de turbidez medidos en sistemas de monocultivos y policultivos.

\section{Amonio y Nitrito}

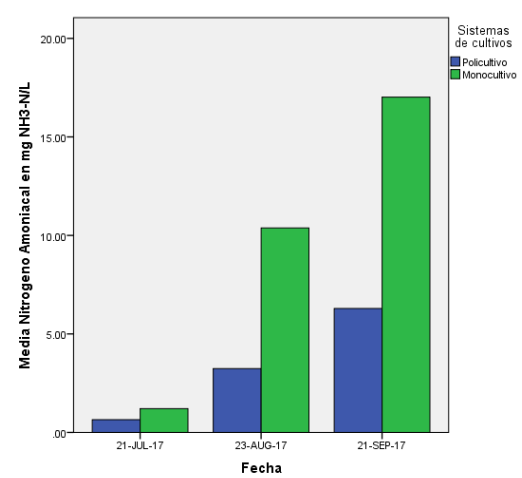

Figura No. 9: Datos de Nitrógeno Amoniacal medidos en mg y tomados en sistemas de policultivo y monocultivo.

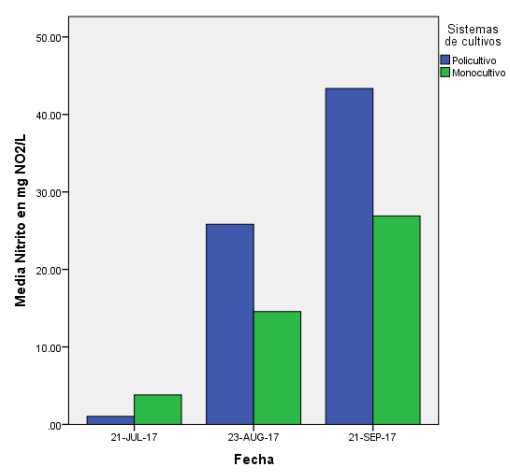

Figura No. 10: Datos de Nitrito medidos en mg y tomados en sistemas de monocultivos y policultivos.

Los valores de nitrógeno amoniacal difieren notablemente entre ambos sistemas de cultivo, siendo el monocultivo el que muestra los valores más altos de amonio a lo largo del ciclo, presentándose valores de $17.02 \mathrm{mgNH}_{3}-\mathrm{N} / \mathrm{L}$ para el monocultivo y $6.29 \mathrm{mgNH}_{3}-\mathrm{N} / \mathrm{L}$ para el policultivo como resultados finales.

Dado esto, puede decirse, que el notable aumento de este parámetro, en ambos sistemas de cultivo, puede atribuirse a los desechos excretados por los organismos 
cultivados y al incremento o acumulación de la materia orgánica dentro del dispositivo, en concordancia con lo descrito por (Boyd, 1996). Aunque en ambos sistemas de cultivo la incidencia de este parámetro se muestra en aumento, para el sistema de policultivo el dato es menor, ya que de acuerdo con (Ruiz, Ibáñez, \& Cacerez, 2007), el pepino de mar (Isostichopus fuscus) posee hábitos alimenticios inclinados al consumo de detritus o materia orgánica, lo que no permitió la descomposición total de la misma, evitando de esta manera una mayor acumulación del amonio dentro del dispositivo.

En cuanto a los valores de nitrito presentes en la figura 10, se puede determinar una notable desigualdad en ambos sistemas de cultivo, (monocultivo y policultivo), obteniendo valores de $43.35 \mathrm{mg}$ No2 -IL para el policultivo y $26.9 \mathrm{mg}$ No2 -IL para el monocultivo, como resultados finales, notándose de esta manera el incremento de este parámetro para el sistema de policultivo en relación con el sistema de monocultivo. De acuerdo a lo descrito por (Sosa, Ramos, Rodriguez, \& Rojas, 2015), la importancia ecológica del pepino de mar (Isostichopus cuscús), radica en la filtración de sedimentos y a la devolución de nutrientes a los procesos de la red alimentaria; por lo que el incremento del nitrito se atribuye a la interferencia de este organismo en el proceso de amonificacion, ya que al filtrar o consumir la materia orgánica se reducen los niveles de nitrógeno amoniacal, como se muestra en la figura (9), pero se permite que el nitrito transformado durante este proceso sea acumulado en mayores cantidades dentro del estanque de policultivo.

\section{Nitrato}

En relación con los datos presentes en la figura 11, se observa una diferencia significativa entre ambos sistemas de cultivo (monocultivo y policultivo) durante el periodo de estudio, notándose una curva de aumento para el sistema de monocultivo y una de descenso para el policultivo, obteniendo como resultados finales, valores de 33.87 $\mathrm{mg} \mathrm{NO} 3 / \mathrm{L}$ para el monocultivo y $18.4 \mathrm{mg}$ No3/L para el policultivo.

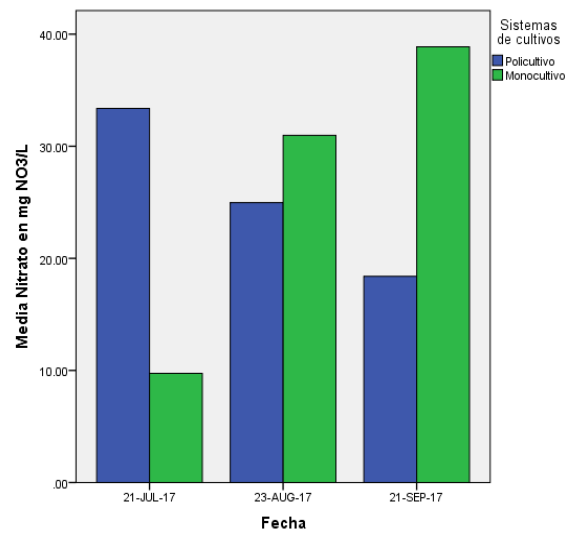

Figura No. 11: Datos de Nitrato medidos en mg y tomados en sistemas de monocultivos y policultivos. 
En los datos presentados en la figura (10) se pueden apreciar valores altos de Nitrito en el sistema de policultivo, por lo que al seguir la secuencia del ciclo del nitrógeno se esperaría que todo ese nitrito acumulado fuese convertido a nitrato durante el proceso de nitrificación, pero al ser el pino de mar un organismo consumidor de fitoplancton al igual que la tilapia, puede atribuirse el descenso del nitrato, en el sistema de policultivo, al consumo de este nutriente por parte de las micro algas, ya que (Velasco, 2003), menciona que algunas de las especies de fitoplancton necesitan una fuente de Nitrógeno como nutriente para su desarrollo, es por ello se puede decir que las micro algas utilizaron el nitrato como fuente de nitrógeno para mantener su curva de crecimiento.

\section{Dinámica de micro algas}

Los conteos de micro algas realizados en ambos sistemas de cultivo (monocultivo y policultivo) presentan diferencias significativas entre ambos, siendo el sistema de monocultivo el que presenta los mayores datos de micro algas encontradas en el dispositivo, siendo en la semana 6 donde se observan los mayores datos de conteo para ambos sistemas de cultivo.

Si bien se observa en la figura (12), en el sistema de monocultivo, el número de micro algas se presenta con mayores valores a partir de la segunda semana de cultivo, en relación al sistema de policultivo, esto es debido a que como se menciona anteriormente, dentro del sistema de policultivo se tienen organismos filtradores que presentan características alimenticias orientadas al consumo de micro algas principalmente, esto descrito por (Ruiz, Ibáñez, \& Cacerez, 2007), es por tal razón que se puede decir que la acción realizada por los pepinos de mar al consumo de micro algas fue la que permitió que el número de micro algas dentro del dispositivo con policultivo se mantuviera dentro de un rango estable durante el periodo de estudio.

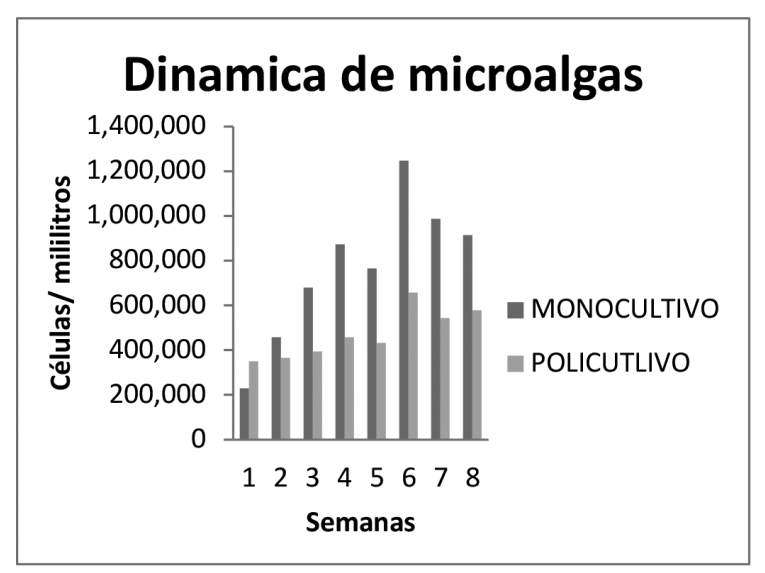

Figura No. 12: Conteo de micro algas, tomados en sistemas de monocultivos y policultivos. 


\section{Factor de conversión alimenticia}

Las evoluciones del factor de conversión alimenticia dentro de ambos sistemas de cultivo se encuentran estrechamente cercanos entre valores siendo el policultivo quien se muestra ligeramente por encima de los valores del monocultivo, durante las primeras 4 semanas experimentales, posiblemente por la gran presencia de micro algas dentro del estanque, como refiere el esquema anterior figura (12). Siendo así la semana 9 la que muestra el mayor índice de conversión alimenticia, para finalizar así de la misma manera tanto el monocultivo, como el policultivo, con un índice de conversión alimenticia de 1,3 gr.

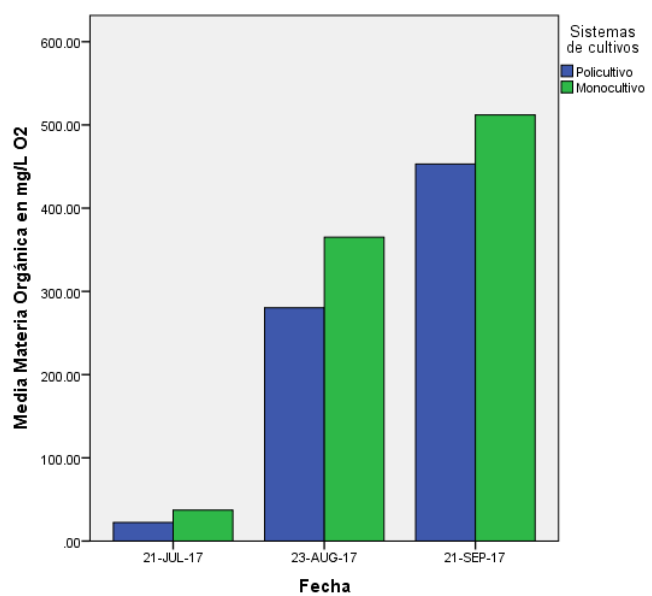

Figura No. 13: Datos de conversión alimenticia medidos en gr y tomados en sistemas de monocultivos y policultivos.

\section{Materia orgánica}

Los valores de materia orgánica reflejados en la figura (12), muestran valores diferenciales entre ambos sistemas de cultivos (monocultivo y policultivo), evidenciándose claramente el aumento de la materia orgánica durante el período de estudio, siendo el sistema de policultivo el que presenta las medias más bajas durante el mismo, finalizando policultivo con una media de $453.0 \mathrm{mg} / \mathrm{L}$ o2 y el monocultivo con $512 \mathrm{mg} / \mathrm{L}$ 02 haciéndose evidente la diferencia entre ambos.

Si bien es notorio el aumento de la materia orgánica en ambos sistemas de cultivo, cabe mencionar que puede atribuirse al incremento de los desechos de los organismos cultivados, debido al aumento de tamaño de estos durante el proceso de cultivo, así como también al desperdicio del alimento no consumido por parte de las tilapias. Por otro lado, el sistema de policultivo con pepino de mar, aunque se muestra en aumento, presenta valores menores en relación con el monocultivo. (Sosa, Ramos, Rodriguez, \& Rojas, 2015) Sugieren que las especies pequeñas de pepino de mar son filtradores 
muy activos y que un individuo de $30 \mathrm{~cm}$ puede procesar $120 \mathrm{~g}$ de sedimento diario, aunque en este caso no se comprueba esta teoría en su totalidad debido a los requerimientos del medio en el que se encontraban si se demuestra la efectividad del pepino sobre la materia orgánica.

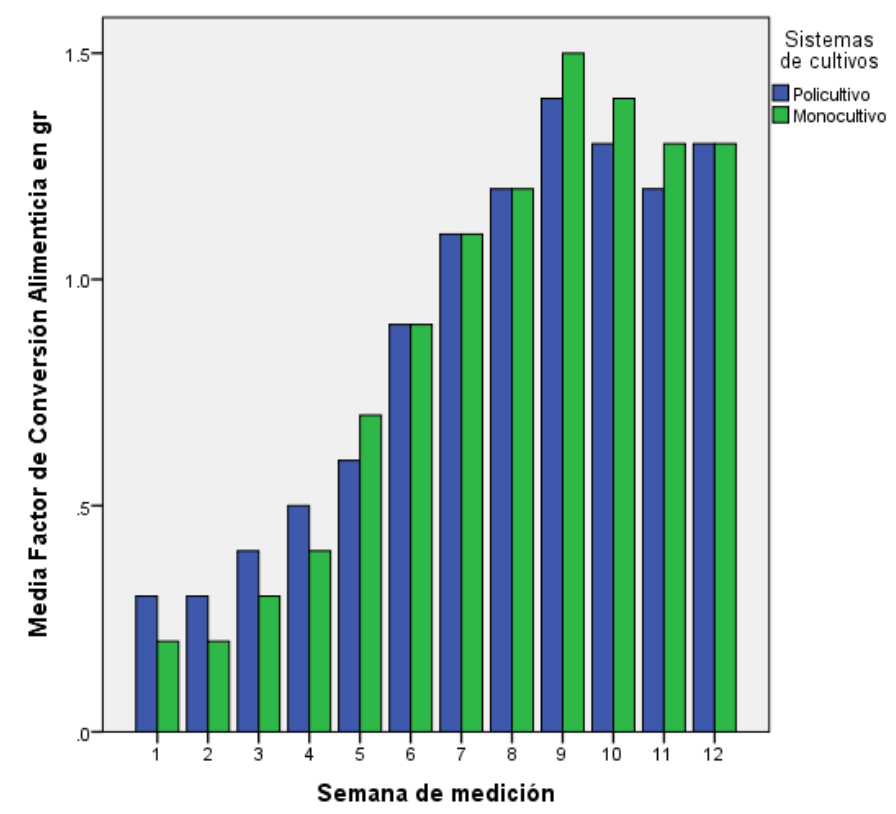

Figura No. 14: Datos de materia orgánica tomados en sistemas de monocultivos y policultivos.

\section{Materia en suspensión}

Según los datos reflejados en la figura (15), se puede decir que hubo diferencia significativa entre ambos sistemas de cultivo (monocultivo y policultivo), siendo el sistema de monocultivo el que presenta el mayor dato de material en suspensión encontrado en este dispositivo, dejando evidenciado la drástica diferencia con el sistema de policultivo, mostrando este un valor diminutivo de $0.32 \mathrm{mg} / \mathrm{lt}$ en relación al sistema de monocultivo el cual presenta $4.48 \mathrm{mg} /$ lt de materia en suspensión.

Como es mencionado ya por (Sosa, Ramos, Rodriguez, \& Rojas, 2015), los pepinos de mar son organismos capaces de remover grandes cantidades de sedimento en el ambiente en que se encuentran, es por ello que en relación a lo antes dicho, la notable disminución del material en suspensión entre ambos sistemas de cultivo, es atribuido a las características de filtración del pepino de mar sobre la materia orgánica, quedando evidenciado una vez más al igual que en la figura (12) la efectividad de filtración q posee el pepino de mar. 


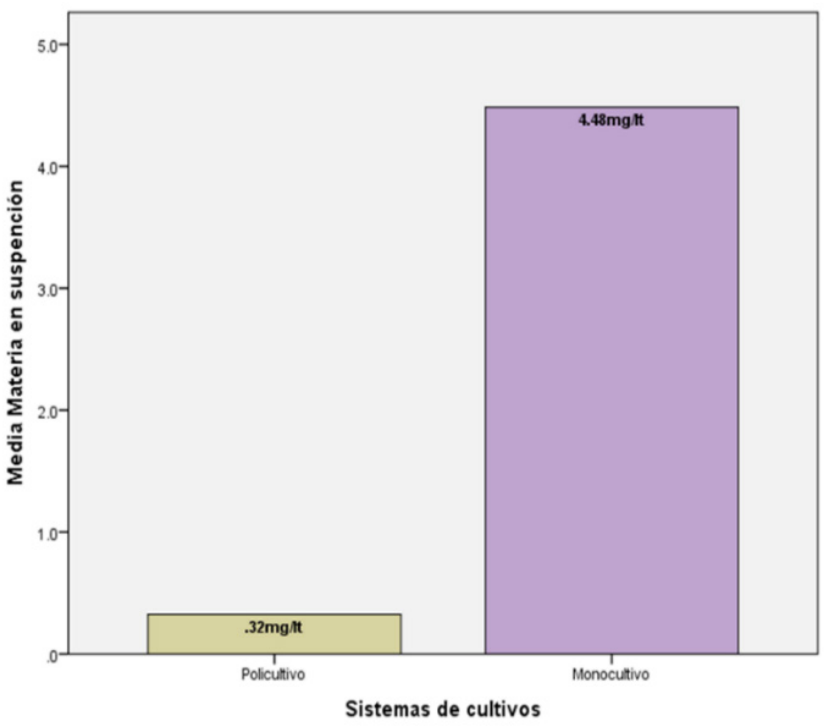

Figura No. 15: Datos de materia en suspensión tomados en sistemas de monocultivo y policultivo.

\section{Conclusiones}

El oxígeno disuelto se obtuvo una media de $4.8 \mathrm{mg} / \mathrm{lt}$, con un valor mínimo durante el cultivo de $0.6 \mathrm{mg} / \mathrm{lt}$ y un máximo de $9 \mathrm{mg} / \mathrm{lt}$, la temperatura tuvo una media de $28.4^{\circ} \mathrm{C}$, con un mínimo de $25^{\circ} \mathrm{C}$ y un máximo de $31^{\circ} \mathrm{C}$, pH se obtuvo una media de 7.8 $\%$, con un mínimo de $6.8 \%$ y un máximo de $9.2 \%$, la salinidad tuvo una media de 31.4 ppm, con un mínimo de $25 \mathrm{ppm}$ y un máximo de $37 \mathrm{ppm}$, la turbidez presento una media de $47.5 \mathrm{~cm}$, con un mínimo de $30 \mathrm{~cm}$ y un máximo de $70 \mathrm{~cm}$., Los niveles de amonio ( $\mathrm{NH}_{4}$ ), nitrito $\left(\mathrm{NO}_{2}\right)$ y nitrato $\left(\mathrm{NO}_{3}\right)$ valorados en ambos sistemas de cultivo presentaron diferencias significativas, siendo el sistema de policultivo quien refleja el mejor comportamiento durante el período de cultivo.

Basado en biometrías realizadas en los sistemas de monocultivo y policultivos con tilapia gris, (Oreochromis sp) se obtienes valores similares con respecto al peso, crecimiento y factor de conversión alimenticia. Basado en los monitoreos de microalgas realizados en los sistemas de monocultivos y policultivos con tilapia gris, (Oreochromis $\mathrm{sp})$ se presentan diferencias significativas entre ambos cultivos, siendo el sistema de monocultivo quien refleja un mayor número de micro algas presentes en el dispositivo. Al realizar los análisis estadísticos se obtuvieron diferencias significativas en los valores de materia orgánica entre ambos sistemas de cultivo, por lo que se considera que la adición de pepinos de mar, como un organismo filtrador en los cultivos de tilapia gris, actúa como un agente de biorremediación teniendo un efecto positivo en cuanto a la disminución de los niveles de materia orgánica en el estanque de cultivo, en relación con un monocultivo de la misma especie. 


\section{Agradecimiento}

Esta publicación obtuvo el financiamiento de: El Fondo de Asistencia Internacional de los Estudiantes y Académicos Noruegos, (SAIH).

\section{Lista de referencia}

Boyd, C. (1996). Potencial del Nitrato de sodio para mejorar las condiciones ambientales de las piscinas de Acuicultura. Recuperado el 12 de 08 de 2017, de http://www. powershow.com/view/2805e8-Zjk1M/Diapositiva_1_flash_ppt_presentation

Bunt, C. M., Mac lsaac, H. J., \& Sprules, W. G. (1993). Pumping Rates and Projected Filtering Impacts of Juvenile Zebra Mussels (Dreissena polymorpha) in Western Lake Erie. Canadian Journal of Fisheries and Aquatic Sciences., 1017-1022. . Recuperado el 19 de 09 de 2016, de http://www.nrcresearchpress.com/doi/ abs/10.1139/f93-117\#.V-dA5YjhDIU

Citrado por Meyer, D. (2004). Introducciòn a la Acuacultura. (Z. Escuela Agricola Panamericana, Ed.) CEIBA, 30(2), 144. Recuperado el o9 de o6 de 2017, de https://bdigital.zamorano.edu/bitstream/11036/2490/1/208986_0363\%20 -\%20Copy.pdf

FONDEPES, F. N. (2004). Mnanual de Cultivo de Tilapia. Lima perú. Recuperado el 13 de 08 de 2017, de http://www2.produce.gob.pe/RepositorioAPS/3/jer/ ACUISUBMENU4/manual_tilapia.pdf

Mortera, E. D. (2013). Biorremediación de efluentes de la Camaronicultura. Repositorio institucional de la universidad Veracruzana., o8. Recuperado el 21 de o9 de 2016, de http://cdigital.uv.mx/bitstream/123456789/33929/1/morteranavaeduardo.pdf

Olivera, A., \& Brito., L. O. (2005). Treating shrimp farming effluent using the native oyster, Crassostrea rhizophorae, in Brazil. researchgate, 60-63. . Recuperado el 19 de o9 de 2016, de https://www.researchgate.net/profile/Luis_Brito5/ publication/257881286_Treating_shrimp_farming_effluent_using_the_native_oyster_Crassostrea_rhizophorae_in_Brazil/links/oob7d5322054744b530 ooooo.p

Pérez, A. A., Bejarano, J. B., \& Barragan, J. J. (2013). Construcción de un Sistema de Instrumentación para la Medicion de la Temperatura, $\mathrm{pH}$ y Oxigeno Disuelto presentes en la Piscicultura bajo Condiciones de Estanques Artificial. Scientia de Tecnica Año XVIII, 18(2), 401-408. Recuperado el 14 de o8 de 2017, de http://www.scielo. org.co/scielo.php?pid=So120-29522011000200002\&script=sci_arttext\&tlng=en 
Peralta, M. C. (2010). Manifestaciòn del impacto Acuicola-Pesquero, Reproducción de pepino de mar (Isostichopus Fuscus) EN LABORATORIO. (S. CUCUMBERMEX, Ed.) semarnat, 83. Recuperado el 2017 de 05 de 11, de http://sinat.semarnat. gob.mx/dgiraDocs/documentos/sin/estudios/2010/25SI2010PDo69.pdf

Ruiz, F., Ibáñez, C., \& Cacerez, C. (2007). Morfometría del tubo digestivo y alimentación del pepino de mar Athyonidium chilensis (Semper, 1868) (Echinodermata: Holothuroidea). Revista de Biología Marina y Oceanografía., 42(3), 269-274. Recuperado el 13 de 08 de 2017

Sosa, A., Ramos, J., Rodriguez, R., \& Rojas, I. (2015). Caracterización del contenido del tracto digestivo del pepino de mar holothuria floridana (Pourtales, 1851) en el litoral de Campeche, México. ResearchGate, 26(1), 1-9. Recuperado el 14 de 08 de 2017, de https://www.researchgate.net/profile/R_Rojas-Gonzalez/ publication/284550668_Caracterizacion_del_contenido_del_tracto_digestivo_del_pepino_de_mar_holothuria_floridana_Pourtales_1851_en_el_litoral_de_Campeche_Mexico/links/5654bda508ae1ef92976dc24.pdf

Sandra Pardo, H. S. (2006). Tratamiento de efluentes:una vía para la acuicultura responsable. Revista MVZ Córdoba., Vol. N¹1, 20-29. Recuperado el 16 de o9 de 2016, de https://dialnet.unirioja.es/servlet/revista?codigo=12779

Soto, D., \& Mena Guillermo, F. d. (1999). Filter feeding by the freshwater mussel, Diplodon chilensis, as a biocontrol of salmon farming eutrophication. Aquaculture, Moritz Botany., 65-81. Recuperado el 19 de og de 2016, de http://www.sciencedirect. com/science/article/pii/Soo44848698004207

Tacon, A., \& Forster, I. P. (2003). Aquafeeds and the environment: policy implications. En A. Tacon, \& I. P. Forster, Management of Aquaculture Effluents. (págs. 181189). Amsterdam: Elsevier, Amsterdam, PAYS-BAS (1972) (Revue). Recuperado el 23 de o9 de 2016, de http://www.sciencedirect.com/science/article/pii/ Soo 44848603004769

USAID, U. S. (2007). INFORME TÉCNICO ENSAYO DE CULTIVO DE TILAPIA (Oreochromis niloticus) EN ESTANQUES DE CAMARÓN. Managua-Nicaragua.: Universidad Centro Americana, Centro de Ivestigaciones de Sistemas Acuaticos. Recuperado el 2017 de 05 de 11, de http://pdf.usaid.gov/pdf_docs/Pnadk646.pdf

Velasco, R. (2003). The algal community as an indicator of the trophic status of lake Patzcuaro, México. Mexico: Environmental Pollution. Recuperado el o3 de o9 de 2017, de http://www.inecc.gob.mx/descargas/cuencas/cong_nal_o6/ tema_05/17_magdalena_velazquez.pdf 